\title{
Perceptions of fecal microbiota transplantation for Clostridium difficile infection: factors that predict acceptance
}

\author{
Leslie Parka , Anjali Mone ${ }^{a}$, Jennifer C. Price ${ }^{b}$, Demetrios Tzimasa, Jacqueline Hirsh ${ }^{a}$, Michael A. Poles ${ }^{a}$, \\ Lisa Maltera, Lea Ann Chen ${ }^{a}$
}

NYU Langone Medical Center, NY; University of California, San Francisco, USA

\section{Abstract}

${ }^{\mathrm{a} D i v i s i o n}$ of Gastroenterology, NYU Langone Medical Center, New York City, NY (Leslie Park, Anjali Mone, Demetrios Tzimas, Jacqueline Hirsh, Michael A. Poles, Lisa Malter, Lea Ann Chen); ${ }^{\mathrm{b} D i v i s i o n}$ of Gastroenterology, University of California San Francisco, San Francisco, California (Jennifer C. Price), USA

Conflict of Interest: None

Correspondence to: Lea Ann Chen, MD, 462 First Ave, 10E1, New York, NY 10016, USA, Tel.: +1 212263 3095,

Fax: +1 844729 1952, e-mail: leaann.chen@nyumc.org

Received 22 June 2016; accepted 16 September 2016; published online 20 October 2016

DOI: https://doi.org/10.20524/aog.2016.0098

\section{Introduction}

Clostridium difficile (C. difficile) are spore-forming anaerobic bacteria [1]. Infection with toxigenic strains of this organism can cause a range of effects on its human hosts from asymptomatic carriage to life-threatening colitis. Although C. difficile-associated diarrhea (CDAD) was traditionally thought to primarily affect elderly, hospitalized, or chronically ill patients, the worldwide incidence of community-acquired $\mathrm{CDAD}$ has increased substantially in recent years [2-6]. CDAD can be transmitted not only by those with the illness, but probably also through asymptomatic carriers and environmental reservoirs [2]. Furthermore, there is a rising number of severe cases refractory to traditional antibiotic therapy, which has contributed to the increase 
in C. difficile-related mortality [7-9]. In recent years, CDAD has become an increasingly common cause of hospitalization, contributing to the burden of rising healthcare costs $[9,10]$.

Fifteen to thirty percent of CDAD patients who initially respond develop recurrent disease, and $65 \%$ of those patients are destined to develop more than one recurrence [11-13]. Therapies for recurrent CDAD include antibiotic tapers, pulsed antibiotic dosing, anion exchange resins, and administration of intravenous immunoglobulins or monoclonal antibodies; only limited data are available for all these therapies [14]. FMT is the latest treatment option for recurrent CDAD infections in patients who have previously failed traditional therapies, with a cure rate of approximately $90 \%[15,16]$. During FMT, a liquid suspension of healthy donor stool is transplanted into an affected patient's gastrointestinal tract through nasogastric tube, enema, or colonoscopy. While the specific mechanism by which FMT treats CDAD is unknown, several studies have suggested that re-establishment of a diverse gut microbiome is key [17-19]. Other studies in mice and humans have shown that treatment with a limited number of gut bacteria can also cure $\mathrm{CDAD}$, suggesting that transplantation of certain microbial functions, rather than merely diversity, is important [20-22].

There are now various case series and one randomized control study describing over 500 patients with resistant CDAD [23] treated with FMT [16]. While short-term adverse effects are limited $[15,16]$, the long-term safety of FMT is still largely unknown. Notably, at the time of writing, the United States Food and Drug Administration has endorsed enforcement discretion for those providing FMT only for C. difficile-related indications, though there is no clinical indication for which FMT is currently FDA-approved [24].

As with any therapy, uptake of a new treatment modality depends not only on its efficacy, but also on physicians' and patients' perceptions of the therapy. Given the unsavory concept of administering donor stool into the colon of a recipient, patient and practitioner apprehension may constitute barriers to the uptake of FMT. Currently, only a few studies have examined the attitudes of the general patient population towards FMT. While these studies are important first steps in understanding the ultimate acceptance of this emerging therapy, the patients studied were limited to single medical centers, those who had already undergone FMT, or those considering FMT for non-CDAD indications $[15,25,26]$. Since the results of these studies may not reflect the attitudes of a broader patient population, we developed a survey to assess the interests and concerns regarding FMT for CDAD that was distributed to patients from varied ethnic and socioeconomic backgrounds. To our knowledge, this is the largest and most diverse study to examine patients' perceptions of FMT.

\section{Materials and methods}

\section{Survey development}

We designed a survey in the English language to evaluate perceptions of FMT and barriers to its recognition as an acceptable therapeutic option (see Appendices). The survey included the following sections: 1) demographic data; 2) experience with complementary and alternative medications (CAM) and therapies; 3) personal medical history; 4) personal history of chronic diarrhea; 5) prior knowledge/experience of CDAD; 6) an introduction and description of FMT; and 7) an assessment of attitudes towards FMT and FMT delivery mode. The questions were geared toward discovering factors that may influence perceptions of FMT, in addition to general demographic data that may be predictive of acceptance of FMT.

\section{Study participants and recruitment}

Surveys were distributed over a 10 -month period in the waiting areas of adult gastroenterology clinics of an urban public hospital (Bellevue Hospital Center), an urban Veterans Affairs hospital (New York Harbor Healthcare SystemManhattan Campus), and in faculty practices affiliated with New York University's Langone Medical Center, all located in New York City. Surveys were completed by and collected from patients waiting for their appointments. Participation was voluntary and anonymous; the participants were not compensated. Review board approval was waived at all 3 participating hospitals.

\section{Statistical analysis}

Statistical calculations were performed using STATA $^{\mathrm{TM}}$ 12.1 software (StataCorp). Unanswered questions were omitted from the analysis. Pearson $\chi^{2}$ analysis was used to evaluate univariate factors associated with FMT acceptance. Factors that accounted for FMT acceptability with $\mathrm{P}<0.10$ on univariate analysis were analyzed by multivariable logistic regression. In addition, the existence of either first- or secondhand experience with $C$. difficile infection was also included in our model, given its presumed influence on FMT acceptability. For all analyses, a P-value $<0.05$ was considered statistically significant.

\section{Results}

A total of 267 adult patients were surveyed across all clinical sites (Table 1). Overall, 12\% (29/234) of respondents were aware of FMT prior to the survey and 77\% (163/213) of all of the subjects would undergo the procedure if medically indicated. Twenty-nine percent knew of $C$. difficile before the survey, with $4 \%$ reporting a personal history of CDAD. Eight percent of patients reported a friend or family member who had suffered from the illness. Nine percent reported a personal history of chronic diarrhea.

Fifty-six percent of respondents reported a history of CAM use (such as acupuncture, Ayurvedic medicine, colonics, chiropractic medicine, homeopathy, or traditional Chinese 
Table 1 Demographics of survey participants

\begin{tabular}{lc}
\hline Sites & \\
VA clinic & $50 \%(133 / 267)$ \\
Faculty group practice & $28 \%(75 / 267)$ \\
Public hospital & $22 \%(59 / 267)$ \\
Male & $65 \%(170 / 260)$ \\
English as primary language & $88 \%(229 / 261)$ \\
\hline Race (self-identified) & \\
White & $54 \%(139 / 256)$ \\
Black & $23 \%(59 / 256)$ \\
Asian & $7.8 \%(20 / 256)$ \\
Other & $14 \%(38 / 256)$ \\
Education & \\
Did not complete high school & $7.5 \%(20 / 266)$ \\
High school & $26 \%(68 / 266)$ \\
Vocational school & $4.5 \%(12 / 266)$ \\
College & $40 \%(105 / 266)$ \\
Graduate school & $23 \%(61 / 266)$ \\
Income (K/year) & \\
$<25$ & $40 \%(94 / 238)$ \\
$25-49$ & $27 \%(63 / 238)$ \\
$50-74$ & $18 \%(42 / 238)$ \\
\hline Kave children $\$ 1000$ & $5.9 \%(14 / 238)$ \\
\hline
\end{tabular}

medicine). Thirty-two percent of respondents reported current or prior use of probiotics, half through pill formulations and half through yogurts. Of those using probiotics, $5 \%$ did so as therapy for chronic diarrhea and $26 \%$ for overall health.

On univariate analysis, subjects with children and those with at least a university degree were more likely to agree to FMT ( $81 \%$ vs. $69 \%, \mathrm{P}=0.04 ; 81 \%$ vs. $68 \%, \mathrm{P}=0.03$, respectively). Other factors, including a history of chronic diarrhea, use of CAM or probiotics, or prior experience with $C$. difficile infection, were not found to be significantly associated with FMT acceptability. There was a trend towards willingness to undergo FMT among those who were married versus unmarried ( $85 \%$ vs. $73 \%, \mathrm{P}=0.06$ ).

On multivariable analysis, we accounted for variables found to be significant at a $\mathrm{P}<0.10$ level on univariate analysis; these included having children, being married, and having at least a college level education. In addition, we accounted for first or second hand experience with C. difficile infection in our model. Again, respondents with children and those with college degrees or higher were more likely to accept FMT (odds ratio [OR] 2.11, 95\% confidence interval [CI] 1.02-4.35, P=0.04; OR 2.27, 1.11-4.60, $\mathrm{P}=0.02$, respectively) (Table 2).

Our survey, which included a brief primer on FMT delivery modalities, also evaluated which modalities would be preferred by patients. When asked to rank FMT delivery by nasogastric tube (NGT), enema, or colonoscopy, $71 \%$ of respondents ranked colonoscopy as the most acceptable vehicle for stool transplant, while NGT was the least preferred.

We then evaluated possible sources of the negative perceptions of FMT. Disease transmission was the most commonly cited concern $(30 \%, 72 / 242)$. Other concerns included fear of the delivery procedure required for FMT (21\%, $50 / 242)$ and the "dirty/unsanitary" perception of FMT (22\%, $53 / 241)$. The success rate of the fecal transplant was the least selected concern (9.1\%) (Fig. 1).

\section{Discussion}

FMT is an effective treatment for recurrent $\operatorname{CDAD}[15,16]$. However, as with other procedures, such as screening colonoscopies, effectiveness may be tempered by patient acceptance [27]. There are currently few studies in the literature that evaluate patient perceptions of this emerging therapy. Our study expands upon this literature in both the number and breadth of the patients evaluated, as it was conducted across clinic sites for 3 distinct hospitals that together serve patients from a large range of socioeconomic, educational, and cultural backgrounds. Furthermore, we explored the factors that motivate acceptance or rejection of this therapy.

Our results show that, despite its inherently unappealing aspects, most patients are open to FMT as a therapeutic option for recurrent CDAD. This is similar to the findings of Kahn et al [29], who published the first study investigating patient attitudes towards FMT, evaluating it in the context of treating ulcerative colitis (UC). Using focus groups, the investigators interviewed 15 adults with UC and 7 parents of children with UC and found them willing to pursue FMT for themselves or their child as an adjunct to standard UC treatments. Interestingly, in another study of UC patients $(n=95)$ by Kahn et al [26], the majority were willing to consider FMT despite satisfactory disease control with their current conventional treatments. In fact, patients identified safety and efficacy as the most important factors in choosing FMT as a therapeutic option, followed by the need for surgery and their physician's recommendation. As expected, patients with previous hospitalizations for UC and severe disease showed a greater interest in FMT. In our study, FMT efficacy was the least selected concern regarding the therapy. While this may be appropriate regarding FMT for CDAD indications, it is worth noting that early efficacy data on FMT for other diseases, while promising, are far more modest [28-30]. In line with such findings, at the time of this writing, the Federal Drug Administration is exercising enforcement discretion regarding Investigational New Drug (IND) applications for the clinical use of FMT for C. difficile not responding to standard therapies [31]. However, an IND is required for all other indications.

Also in 2012, Zipursky et al [25] utilized structured surveys, including hypothetical case scenarios, to investigate perceptions of FMT for recurrent CDAD in general medicine patients, the majority of whom had not suffered from CDAD 
Table 2 Logistic regression analyses of variables affecting FMT acceptability

\begin{tabular}{lll}
\hline Respondents with children & $1.95(\mathrm{P}=0.04,1.02-3.69)$ & $2.11(\mathrm{P}=0.04,1.02-4.35)$ \\
Married & $2.04(\mathrm{P}=0.07,0.95-4.39)$ & $1.54(\mathrm{P}=0.30,0.68-3.46)$ \\
College or graduate education & $2.08(\mathrm{P}=0.03,1.09-3.95)$ & $2.27(\mathrm{P}=0.02,1.11-4.60)$ \\
\hline Experience with C. difficile & $1.57(\mathrm{P}=0.22,0.758-3.24)$ & $1.48(\mathrm{P}=0.32,0.68-3.22)$ \\
\hline
\end{tabular}

${ }^{*}$ Either personally or through a friend or relative. While Clostridium difficile (C. difficile) experience was found to have a P value $>0.10$ on univariate analysis, it was included in our multivariable regression analysis because of its presumed influence on FMT acceptability

FMT, fecal microbiota transplantation; CI, confidence interval

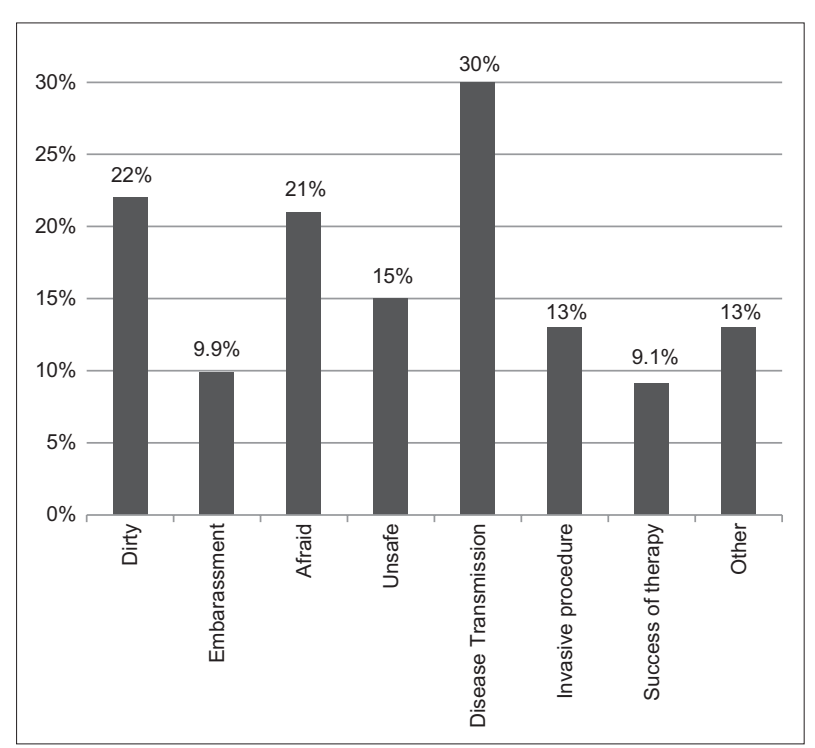

Figure 1 Percentage of patients expressing concerns about FMT perceptions

or had the procedure previously. In this single-center study, the majority of subjects were white $(93.5 \%)$ and female (70\%). In contrast, our respondents included 23\% black, 27\% Hispanic, and $7.8 \%$ Asian patients, reflecting our large metropolitan environment. Our study was also predominantly male (65\%). Regardless of the cultural differences between the two study populations, as well as the growing use of FMT, both studies indicate a lack of knowledge about FMT in the community, with only approximately $10 \%$ of respondents being aware of the therapy prior to survey completion. In addition, both studies found that a majority of individuals would undergo the procedure if medically indicated. Thus, despite the differences in sex and ethnicity distributions, patients' perceptions of stool transplantation are comparable.

Our findings further provide interesting insight into the potential factors that may influence patient acceptance of fecal transplant. On univariate and multivariable analyses, having children was a significant predictor for accepting FMT. It is possible that patients with children are more likely to embrace a treatment that is inherently unappealing in nature because of their drive to provide for their family. This hypothesis is further supported by our results showing a trend toward accepting FMT among those married versus unmarried. A similar impact of familial relationships on the acceptance of medical procedures has also been found in the colon cancer screening literature [32-35]. For example, Kotwal et al found higher screening colonoscopy rates among married versus unmarried men. Also, husbands were more likely to obtain colonoscopies if they described their wives as happy with their marriage, more highly educated, and more supportive [35].

In our study, at least $63 \%$ of patients had university or graduate degrees. This level of education was found to be a significant predictor of FMT acceptance on univariate and multivariable analyses, suggesting that education and, presumably greater health literacy, plays an important role in FMT acceptance. Each of the prior studies of FMT acceptability also identified a positive correlation between higher education level and increased acceptance rates. Prior studies have also shown that knowledge of alternative medicine is correlated with the level of education [36,37]. While the majority of study respondents (56\%) had personal experience with using probiotics or CAM, the use of such therapies did not correlate with FMT acceptance in our study.

One of the barriers to FMT uptake is the "yuck factor" associated with internalizing a filthy product. Though our study respondents were concerned that the administered stool was "dirty" (22\%) and feared the procedure (21\%), the most common patient concern was about disease transmission (30\%). While the current literature does not support any significant disease transmission in FMTs [38], transmission of currently unrecognized infectious agents remains a possibility and is an important area for continued research.

Consideration must also be given to the mechanism of donor stool delivery. Our findings suggest a preference for colonoscopy as the most acceptable vehicle for stool transplant and NGT as the least acceptable. However, there are still practical barriers to these delivery modalities as they are invasive, unpleasant, and have the potential for complications [15]. Studies exploring more esthetically appealing, less invasive and easier methods for administration of FMT are ongoing. In a small pilot study $(\mathrm{n}=20)$, Youngster et al demonstrated $90 \%$ clinical resolution of recurrent CDAD after oral administration of frozen capsules of fecal material from unrelated donors [39]. There were no serious or unexpected adverse events. This new FMT delivery modality may help promote the availability of FMT, as it eliminates the need for patients and medical providers to handle stool suspensions and obviates the need for a gastrointestinal procedure. 
Finally, another barrier to the widespread utilization of FMT to treat complicated CDAD is apprehension amongst physicians [40]. While not addressed in our study, investigation into physician attitudes reveals a lack of knowledge about the procedure itself, a misperception about patient attitudes toward FMT, and an aversion for handling stool during the treatment, with up to $29 \%$ of physicians reporting it was unappealing enough to prevent them from offering this therapy despite positive reported data [41].

Our study has some limitations. Since the survey was only distributed in English, it is difficult to draw conclusions on the views of immigrant patient populations with potential language barriers receiving healthcare in the United States. In addition, the education level and literacy of our patients may have affected their ability to fully complete and understand a somewhat lengthy study survey. Those who had difficulties reading or understanding English, regardless of reason, may have had difficulties understanding certain questions. This may have contributed to the unanswered questions on the survey, though some questions were left unanswered because of time limitations. Finally, our surveys only evaluated patients' attitudes; they did not assess physicians' attitudes toward FMT and their possible contribution to the barriers against FMT utilization.

In conclusion, FMT is an effective treatment modality for recurrent $\mathrm{CDAD}$ and may have significant application in other major diseases, including metabolic syndrome, inflammatory bowel disease, and other autoimmune conditions. Despite limited prior knowledge of FMT and concerns about safety, patients are receptive to the procedure, highlighting a role for shared decision-making when considering this emerging therapy. Attitudes toward FMT are modifiable barriers to

\section{Summary Box}

\section{What is already known:}

- Fecal microbiota transplantation (FMT) is an emerging, effective therapy for recurrent Clostridium difficile infection

- Patients who have previously failed traditional therapies and try FMT exhibit a cure rate of approximately $90 \%$

- Despite its effectiveness, some patients are reluctant to accept FMT treatment

\section{What the new findings are:}

- In this study, most patients had no prior knowledge of FMT, but were receptive to the procedure

- Having children and higher education levels were significant predictors for FMT acceptance

- Our findings suggest that barriers to FMT utilization may be overcome with counseling about safety concerns the wide-scale use of this therapy in the CDAD treatment armamentarium. Furthermore, advancements in the delivery method, such as encapsulation of donor stool, may further improve FMT's safety and acceptability.

\section{Acknowledgment}

We would like to thank Arielle Radin for her technical assistance and manuscript formatting.

\section{References}

1. Ananthakrishnan AN. Clostridium difficile infection: epidemiology, risk factors and management. Nat Rev Gastroenterol Hepatol 2011;8:17-26.

2. Eyre DW, Cule ML, Wilson DJ, et al. Diverse sources of C. difficile infection identified on whole-genome sequencing. $N$ Engl J Med 2013;369:1195-1205.

3. Kuntz JL, Chrischilles EA, Pendergast JF, Herwaldt LA, Polgreen PM. Incidence of and risk factors for communityassociated Clostridium difficile infection: a nested case-control study. BMC Infect Dis 2011;11:194.

4. Khanna S, Pardi DS, Aronson SL, et al. The epidemiology of community-acquired Clostridium difficile infection: a populationbased study. Am J Gastroenterol 2012;107:89-95.

5. Eyre DW, Tracey L, Elliott B, et al. Emergence and spread of predominantly community-onset Clostridium difficile PCR ribotype 244 infection in Australia, 2010 to 2012. Euro Surveill 2015;20:21059.

6. Freeman J, Bauer MP, Baines SD, et al. The changing epidemiology of Clostridium difficile infections. Clin Microbiol Rev 2010;23:529-549.

7. Morris AM, Jobe BA, Stoney M, Sheppard BC, Deveney CW, Deveney KE. Clostridium difficile colitis: an increasingly aggressive iatrogenic disease? Arch Surg 2002;137:1096-1100.

8. Pépin J, Valiquette L, Alary ME, et al. Clostridium difficileassociated diarrhea in a region of Quebec from 1991 to 2003: a changing pattern of disease severity. CMAJ 2004;171:466-472.

9. Zilberberg MD, Shorr AF, Kollef MH. Increase in adult Clostridium difficile-related hospitalizations and case-fatality rate, United States, 2000-2005. Emerg Infect Dis 2008;14:929-931.

10. Steiner C BM, Sun Y, Weiss A. HCUP Projections: Clostridium difficile hospitalizations 2003-2014. 2014. HCUP Projections Report \#2014-03. U.S. Agency for Healthcare Research and Quality https://hcup-us.ahrq.gov/reports/projections/2014-03.pdf Accessed 2016 October 6.

11. McFarland LV, Elmer GW, Surawicz CM. Breaking the cycle: treatment strategies for 163 cases of recurrent Clostridium difficile disease. Am J Gastroenterol 2002;97:1769-1775.

12. McFarland LV, Surawicz CM, Greenberg RN, et al. A randomized placebo-controlled trial of Saccharomyces boulardii in combination with standard antibiotics for Clostridium difficile disease. JAMA 1994;271:1913-1918.

13. McFarland LV, Surawicz CM, Rubin M, Fekety R, Elmer GW, Greenberg RN. Recurrent Clostridium difficile disease: epidemiology and clinical characteristics. Infect Control Hosp Epidemiol 1999;20:43-50.

14. Leffler DA, Lamont JT. Treatment of Clostridium difficile-associated disease. Gastroenterology 2009;136:1899-1912.

15. Brandt LJ, Aroniadis OC, Mellow M, et al. Long-term follow-up of 
colonoscopic fecal microbiota transplant for recurrent Clostridium difficile infection. Am J Gastroenterol 2012;107:1079-1087.

16. van Nood E, Vrieze A, Nieuwdorp M, et al. Duodenal infusion of donor feces for recurrent Clostridium difficile. $N$ Engl J Med 2013;368:407-415.

17. Shahinas D, Silverman M, Sittler T, et al. Toward an understanding of changes in diversity associated with fecal microbiome transplantation based on 16S rRNA gene deep sequencing. MBio 2012;3. doi:10.1128/mBio.00338-12

18. Chang JY, Antonopoulos DA, Kalra A, et al. Decreased diversity of the fecal Microbiome in recurrent Clostridium difficile-associated diarrhea. J Infect Dis 2008;197:435-438

19. Hamilton MJ, Weingarden AR, Unno T, Khoruts A, Sadowsky MJ. High-throughput DNA sequence analysis reveals stable engraftment of gut microbiota following transplantation of previously frozen fecal bacteria. Gut Microbes 2013;4:125-135.

20. Petrof EO, Gloor GB, Vanner SJ, et al. Stool substitute transplant therapy for the eradication of Clostridium difficile infection: 'RePOOPulating' the gut. Microbiome 2013;1:3.

21. Buffie CG, Bucci V, Stein RR, et al. Precision microbiome reconstitution restores bile acid mediated resistance to Clostridium difficile. Nature 2015;517:205-208.

22. Lawley TD, Clare S, Walker AW, et al. Targeted restoration of the intestinal microbiota with a simple, defined bacteriotherapy resolves relapsing Clostridium difficile disease in mice. PLoS Pathog 2012;8:e1002995.

23. Cammarota G, Ianiro G, Gasbarrini A. Fecal microbiota transplantation for the treatment of Clostridium difficile infection: a systematic review. J Clin Gastroenterol 2014;48:693-702.

24. Enforcement Policy Regarding Investigational New Drug Requirements for Use of Fecal MIcrobiota for Transplantation to Treat Clostridium difficile Infection Not Reponsive to Standard Therapies. Guidance for Industry 2013; http:// www.fda.gov/downloads/BiologicsBloodVaccines/ GuidanceComplianceRegulatoryInformation/Guidances/ Vaccines/UCM361393.pdf. Accessed 2016 June 21.

25. Zipursky JS, Sidorsky TI, Freedman CA, Sidorsky MN, Kirkland KB. Patient attitudes toward the use of fecal microbiota transplantation in the treatment of recurrent Clostridium difficile infection. Clin Infect Dis 2012;55:1652-1658.

26. Kahn SA, Vachon A, Rodriquez D, et al. Patient perceptions of fecal microbiota transplantation for ulcerative colitis. Inflamm Bowel Dis 2013;19:1506-1513.

27. Lansdorp-Vogelaar I, Kuntz KM, Knudsen AB, van Ballegooijen M, Zauber AG, Jemal A. Contribution of screening and survival differences to racial disparities in colorectal cancer rates. Cancer Epidemiol Biomarkers Prev 2012;21:728-736.

28. Gimeno García AZ. Factors influencing colorectal cancer screening participation. Gastroenterol Res Pract 2012;2012:483417.

29. Kahn SA, Gorawara-Bhat R, Rubin DT. Fecal bacteriotherapy for ulcerative colitis: patients are ready, are we? Inflamm Bowel Dis 2012;18:676-684.

30. Colman RJ, Rubin DT. Fecal microbiota transplantation as therapy for inflammatory bowel disease: a systematic review and metaanalysis. J Crohns Colitis 2014;8:1569-1581.

31. Moayyedi P, Surette MG, Kim PT, et al. Fecal microbiota transplantation induces remission in patients with active ulcerative colitis in a randomized controlled trial. Gastroenterology 2015;149:102-109.

32. Rossen NG, Fuentes S, van der Spek MJ, et al. Findings from a randomized controlled trial of fecal transplantation for patients with ulcerative colitis. Gastroenterology 2015;149:110-118.

33. Denberg TD, Melhado TV, Coombes JM, et al. Predictors of nonadherence to screening colonoscopy. J Gen Intern Med 2005;20:989-995.

34. van Jaarsveld CHM, Miles A, Edwards R, Wardle J. Marriage and cancer prevention: does marital status and both spouses together influence colorectal cancer participation? J Med Screen 2006; 13:172-176.

35. Kotwal AA, Lauderdale DS, Waite LJ, Dale W. Differences between husbands and wives in colonoscopy use: results from a national sample of married couples. Prev Med 2016;88:46-52.

36. Langhorst J, Anthonisen IB, Steder-Neukamm U, et al. Amount of systemic steroid medication is a strong predictor for the use of complementary and alternative medicine in patients with inflammatory bowel disease: results from a German national survey. Inflamm Bowel Dis 2005;11:287-295.

37. Wong AP, Clark AL, Garnett EA, et al. Use of complementary medicine in pediatric patients with inflammatory bowel disease: results from a multicenter survey. J Pediatr Gastroenterol Nutr 2009;48:55-60.

38. Mullish BH, Marchesi JR, Thursz MR, Williams HR. Microbiome manipulation with faecal microbiome transplantation as a therapeutic strategy in Clostridium difficile infection. QJM 2015;108:355-359.

39. Youngster I, Russell GH, Pindar C, Ziv-Baran T, Sauk J, Hohmann EL. Oral, capsulized, frozen fecal microbiota transplantation for relapsing Clostridium difficile infection. JAMA 2014;312:1772-1778.

40. Brandt LJ. Editorial commentary: fecal microbiota transplantation: patient and physician attitudes. Clin Infect Dis 2012;55:1659-1660.

41. Zipursky JS, Sidorsky TI, Freedman CA, Sidorsky MN, Kirkland KB. Physician attitudes toward the use of fecal microbiota transplantation for the treatment of recurrent Clostridium difficile infection. Can J Gastroenterol Hepatol 2014;28:319-324. 